\title{
Mixed- versus predilution hemodiafiltration effects on convection volume and small and middle molecule clearance in hemodialysis patients: a prospective randomized controlled trial
}

\author{
Kyoung Sook Park ${ }^{1}$, Ea Wha Kang ${ }^{1}$, Tae Ik Chang ${ }^{1}$, Wonji Jo², Jung Tak Park², Tae-Hyun Yoo ${ }^{2}$, Shin-Wook Kang ${ }^{2}$, \\ Seung Hyeok Han $^{2}$ \\ ${ }^{1}$ Department of Internal Medicine, National Health Insurance Service llsan Hospital, Goyang, Republic of Korea \\ ${ }^{2}$ Department of Internal Medicine, Institute of Kidney Disease Research, Yonsei University College of Medicine, Seoul, Republic of Korea
}

Background: The use of newly developed mixed-dilution hemodiafiltration (HDF) can supplement the weaknesses of pre- and postdilution HDF. However, it is unclear whether mixed-HDF performs well compared to predilution HDF.

Methods: We conducted a prospective, open-labeled, randomized controlled trial from two hemodialysis centers in Korea. Between January 2017 and September 2019, 60 patients who underwent chronic hemodialysis were randomly assigned at a 1:1 ratio to receive either predilution HDF $(n=30)$ or mixed-HDF $(n=30)$ for 6 months. We compared convection volume, changes in small- and medium-sized molecule clearance, high-sensitive C-reactive protein (hs-CRP) level, and dialysis-related parameters between the two dialysis modalities.

Results: A mean effective convection volume of $41.0 \pm 10.3 \mathrm{~L} /$ session in the predilution HDF group and $51.5 \pm 9.0 \mathrm{~L} / \mathrm{session}$ in the mixed-HDF group was obtained by averaging values of three time-points. The difference in effective convection volume between the groups was $10.5 \pm 1.3 \mathrm{~L} /$ session. This met the preset noninferiority criteria, suggesting that mixed-HDF was noninferior to predilution HDF. Moreover, the $\beta 2$-microglobulin reduction rate was greater in the mixed-HDF group than in the predilution HDF group, while mixed-HDF provided greater transmembrane pressure. There were no significant between-group differences in Kt/V urea levels, changes in predialysis hs-CRP levels, proportions of overhydration, or blood pressure values. Symptomatic intradialytic hypotension episodes and other adverse events occurred similarly in the two groups.

Conclusion: Use of mixed-HDF was comparable to predilution HDF in terms of delivered convection volume and clinical parameters. Moreover, mixed-HDF provided better $\beta 2$-microglobulin clearance than predilution HDF.

Keywords: Convective volume, Hemodiafiltration, Online hemodiafiltration, Randomized controlled trial

Received: February 22, 2021; Revised: May 30, 2021; Accepted: May 31, 2021

Editor: Young-Ki Lee, Hallym University, Seoul, Republic of Korea

Correspondence: Seung Hyeok Han

Department of Internal Medicine, Institute of Kidney Disease Research, Yonsei University College of Medicine, 50-1 Yonsei-ro, Seodaemun-gu, Seoul 03722, Republic of Korea. E-mail: hansh@yuhs.ac

ORCID: https://orcid.org/0000-0001-7923-5635

Copyright (C 2021 by The Korean Society of Nephrology

(a) This is an Open Access article distributed under the terms of the Creative Commons Attribution Non-Commercial and No Derivatives License (http:// creativecommons.org/licenses/by-nc-nd/4.0/) which permits unrestricted non-commercial use, distribution of the material without any modifications, and reproduction in any medium, provided the original works properly cited. 


\section{Introduction}

Remarkable technical advances have been made with regard to dialysis membranes and hemodialysis (HD) machines in recent decades. In spite of such improvements, mortality rates remain high, and the overall 5-year survival rate in patients of kidney failure with replacement therapy (KFRT) is $30 \%$ in the United States [1]. Unfortunately, dialysis treatment alone cannot improve clinical outcomes, and a comprehensive approach is required. Such an approach should include blood pressure control, appropriate body fluid control, elimination of middle-sized molecules, removal of inflammatory substances, osteoporosis and anemia management, and the provision of sufficient nourishment. Hemodiafiltration (HDF) can facilitate the achievement of these diverse goals [2-5]. In fact, HDF has many advantages over conventional HD, which include its higher clearance of low and middle molecules and inflammatory cytokines, improvement of anemia, and maintenance of hemodynamic stability $[6,7]$. Notably, recent clinical trials have shown that HDF increased patient survival rates compared to conventional HD, especially in patients who received high-volume convective therapy $[7,8]$.

There are two representative HDF modes: pre- and postdilution. Each has strengths and limitations. Postdilution HDF is the most effective way to maximize molecule clearance. However, blood concentrations can be elevated using HDF, which can cause thrombosis. On the other hand, predilution HDF can resolve this problem [9] but requires about three times more purified water than postdilution HDF and does not guarantee maximal clearance. Therefore, the use of mixed-HDF emerged to compensate for the shortcomings of the other two HDF modalities $[10,11]$. In mixed-HDF, the substitution fluid required for dialysis is injected at both the entrance and exit of the dialysis membrane. Using a continuous monitoring and feedback system to assess transmembrane pressure (TMP) in the dialyzer, dialysis is automatically switched to either pre- or postdilution HDF mode and optimizes TMP during HD [12]. Despite these advantages of mixed-HDF, clinical studies testing the efficacy of mixedHDF versus pre- or postdilution HDF are lacking. Several studies have reported that the efficiencies of small- or largesized molecule removal between mixed-HDF and pre- or postdilution HDF are comparable. Also, TMP is maintained at a more stable level in mixed-HDF compared with postdi- lution HDF [13-15]. In parts of East Asia such as Japan, most HD units tend to use predilution HDF due to intrinsic problems with postdilution HDF, including high TMP and the tendency to promote clot formation $[11,16]$. This is also true in Korea, where utilization of predilution HDF is increasing [8]. However, whether mixed-HDF performs well in comparison to predilution HDF remains unknown. Therefore, we conducted a randomized controlled trial to compare predilution HDF with mixed-HDF in Korean patients with KFRT.

\section{Methods}

\section{Study design and participants}

This study was a prospective, randomized, open-label trial conducted at two hospitals in Korea (Severance Hospital, Seoul; National Health Insurance Service [NHIS] Ilsan Hospital, Goyang). Individuals aged 20 to 75 years who had received HD 3 times weekly for $\geq 3$ months were allowed to participate in the study. Exclusion criteria were as follows: (1) life expectancy < 12 months, (2) dialysis treatment received for less than 3 months or initiated due to acute kidney injury, (3) current history of malignancy, (4) pregnancy, (5) contraindication to anticoagulants, (6) systemic blood pressure $<90 \mathrm{mmHg}$, and (7) previously received HDF before enrollment. The study was conducted in accordance with the principles of the Declaration of Helsinki, and the study protocol was approved by the Institutional Review Board at each center (No. 4-2016-0702 for Severance Hospital; No. 2018-11-002 for NHIS Ilsan Hospital). All participants provided informed consent when enrolled in the study.

Between January 2017 and December 2019, a total of 66 patients was screened. After a 1-month screening period, 60 patients were randomly assigned at a 1:1 ratio to receive either mixed-HDF or predilution HDF for 6 months. The random assignment was performed using a web-based, random allocation table that considered institution, sex, and causative disease of KFRT (Fig. 1).

\section{Treatment procedures}

All participants received thrice-weekly dialysis for at least 3 hours. Both groups were treated with the 5008 or $5008 \mathrm{~S}$ dialysis system using CorDiax dialyzer (Fresenius Medical Care, Bad Homburg, Germany) and maintained a blood 


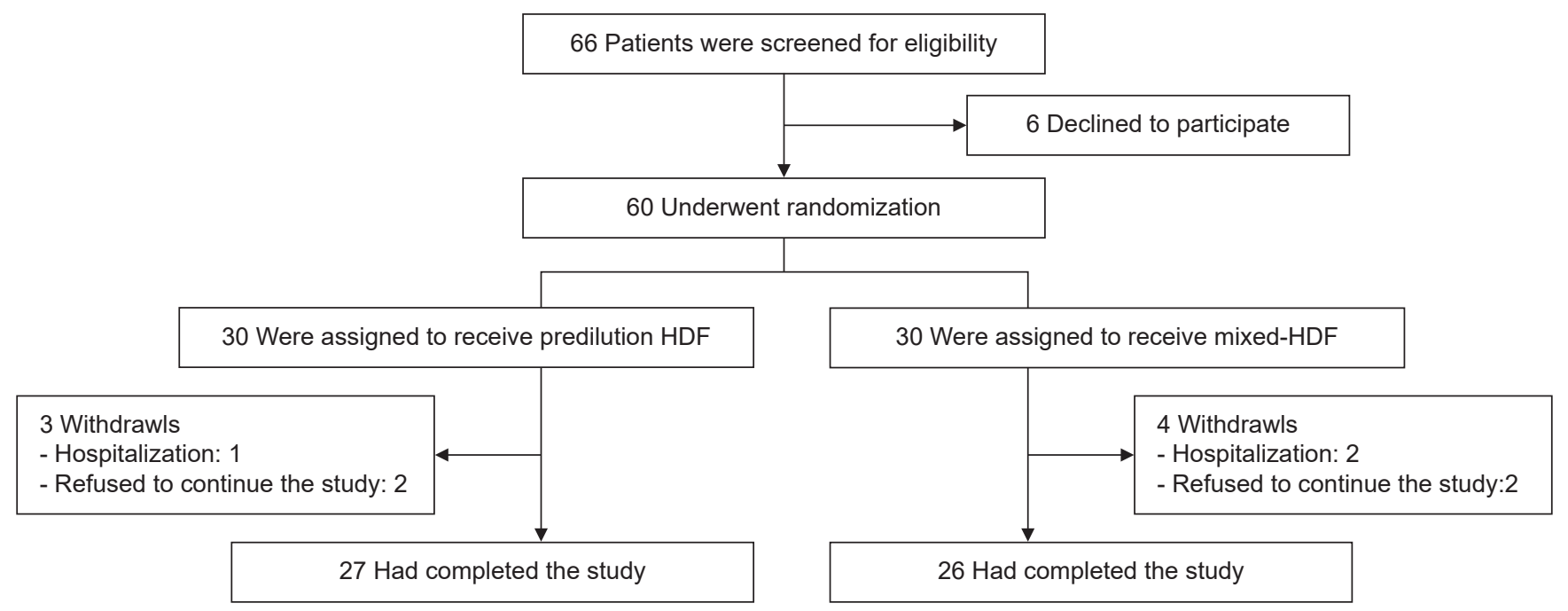

Figure 1. A flow chart of the study population that includes the numbers of patients who were screened, underwent randomization, and completed the assessment.

HDF, hemodiafiltration.

flow rate of $250 \mathrm{~mL} / \mathrm{min}$ and dialysate flow rate of $700 \mathrm{~mL} /$ min. TMP was not expected to exceed $400 \mathrm{mmHg}$ in either predilution HDF or mixed-HDF patients. Prescribed ultrafiltration rate and substitution fluid in both predilution and mixed-HDF groups were calculated using previously reported equations [17].

\section{Study variables}

The demographic and medical history of participants was collected at enrollment. We recorded dialysis-related information at baseline and every 3 months thereafter. Information collected included dialyzer characteristics, dialysis time, dialysis machine, blood and dialysate flows, substitution volume, TMP, height, dry body weight, pre- and postdialysis body weight, delivered convective volume, net ultrafiltration volume, and predialysis systolic and diastolic blood pressure. Further, the following laboratory data were measured at 0,3 , and 6 months after initiation of this research: hemoglobin, hematocrit, white blood cell differential count, platelet count, predialysis urea concentration, creatinine, sodium, potassium, concentration of bicarbonate using total carbon dioxide, calcium, phosphate, intact parathyroid hormone, high-sensitivity C-reactive protein (hs-CRP), albumin, and fasting glucose. To determine the reduction ratio (RR) of $\beta 2$-microglobulin at pre-and postdialysis, serum $\beta 2$-microglobulin level was measured at 0 and 6 months. All laborato- ry tests were performed locally using standard procedures in certified laboratories. The RR of $\beta 2$-microglobulin was calculated using pre- and postdialysis serum $\beta 2$-microglobulin levels and the following formula: $\mathrm{RR}(\%)=[1-$ (concentration of serum $\beta 2$-microglobulin obtained after dialysis/concentration of serum $\beta 2$-microglobulin obtained before dialysis)] $\times 100$. Extracellular fluid and total body water volumes were measured via multiple frequency bioelectrical impedance analysis (BCM; Fresenius Medical Care). Measured extracellular fluid volume is presented as overhydrated (L). Measured relative extracellular fluid volume considering total body water fluid volume is presented as overhydrated-extracellular fluid (\%).

\section{Outcomes}

The primary outcome assessed was the delivered convection volume difference between mixed-HDF and predilution HDF treatment methods [18]. Since the predilution mode of therapy used twice as much replacement fluid as the postdilution mode, we calculated effective convection volume ratios for mixed-HDF as follows: effective convection volume $=$ substitution volume in predilution mode $+2 \times$ substitution volume in postdilution mode + ultrafiltration volume In addition, we compared convection volume adjusted for body surface area between two groups. Secondary outcomes included middle- (RR of $\beta 2$-microglobulin) and small-sized 
molecule clearance (Kt/V urea), changes in predialysis levels of an inflammatory marker (hs-CRP) and phosphate, TMP, blood pressure, and intradialysis tolerance.

\section{Power calculation}

The total substitution fluid volume is always greater when using predilution HDF versus mixed-HDF. Because mixedHDF uses both pre- and postdilution modes during the dialysis session, we hypothesized that the effective convective volume in mixed-HDF would be $120 \%$ of that of predilution $\mathrm{HDF}$, and the difference in convective fluid would be approximately $7.5 \mathrm{~L}$, which was used as a noninferiority limit. Thus, it was determined that 25 patients within each group were needed to detect a 10-L delivered convective volume difference between the two groups assessed with a power of $90 \%$ and an $\alpha$-value of 0.05 . Considering a dropout percentage of $20 \%$, the number needed per group was 30 patients.

\section{Statistical analyses}

All data were analyzed according to the per-protocol principle. Data are expressed as mean \pm standard deviation or as median (range) for skewed data. Baseline clinical data and laboratory findings, measured at the time of random group assignment, were compared using the t-test and chi-square test. In addition, changes in primary and secondary outcome parameters were analyzed using repeated measures analysis of variance (ANOVA). Continuous variables were assessed using a mixed model approach for repeated measurements, after adjustment for age, sex, serum albumin and hemoglobin levels, systolic blood pressure (SBP), predialysis serum $\beta 2$ microglobulin concentration, and dialysis blood flow. A two-sided significance test was used throughout the analysis, and values of $\mathrm{p}<0.05$ were considered significant. All statistical analyses were performed using the STATA version 16 statistical package (StataCorp, College Station, TX, USA).

\section{Results}

\section{Baseline patient characteristics}

The baseline characteristics of patients and treatment parameters are summarized in Table 1. The mean age was 59.7 years, and $50.9 \%$ of study participants were male. All patients had been on dialysis treatment for 2 years (range, 1-4 years). Overall, there were no differences in baseline characteristics observed between the two groups. However, the serum $\beta 2$-microglobulin level of the mixed-HDF group was significantly higher than that of the predilution HDF group. Among dialysis parameters, dialysis time, TMP, dialysis flow rate, and net ultrafiltration did not differ between the two groups. However, the mixed-HDF group had slightly lower blood flow rate than the predilution HDF group.

\section{Primary outcome}

Table 2 and Supplementary Fig. 1A (available online) show convection volumes of the two HDF groups during the 6-month period considered. In the predilution HDF group, the mean convective volume determined by averaging values from three time points was $41.0 \pm 10.3 \mathrm{~L} /$ session, and the convective volume was constantly delivered during follow-up. In the mixed-HDF group, the switch to predilution mode was successfully accomplished depending on TMP level during the dialysis session. In this group, the total effective convection volume delivered was $51.5 \pm 9.0 \mathrm{~L} /$ session in the mixed-HDF group, which was approximately $20 \%$ higher than that of the predilution HDF group. The difference in effective convection volume between the two groups was 10.5 $\pm 1.3 \mathrm{~L} /$ session, which met our preset noninferiority criteria (Fig. 2B, Supplementary Fig. 1B). This finding suggests that mixed-HDF is comparable to predilution HDF with respect to convection volume. Repeated ANOVA analysis and linear mixed model assessment also showed that effective convection volume was greater in the mixed-HDF group than in the predilution HDF group throughout the study period. These results were similar when body surface area-adjusted volumes were compared (Table 2 and Fig. 2).

\section{Secondary outcomes}

In secondary outcome analyses, we first compared middleand small-molecule clearance rates of mixed-HDF and predilution HDF groups. The predialysis serum $\beta 2$-microglobulin level was significantly higher in the mixed-HDF group than the predilution HDF group during the study period. However, serum $\beta 2$-microglobulin level of the mixedHDF group significantly decreased from the baseline value 
Table 1. Baseline characteristics

\begin{tabular}{|c|c|c|c|}
\hline Variable & Predilution HDF $(n=27)$ & Mixed-HDF $(n=26)$ & p-value \\
\hline Age (yr) & $59.0 \pm 1.3$ & $60.5 \pm 1.1$ & 0.63 \\
\hline Male sex & $16(59.3)$ & $11(42.3)$ & 0.22 \\
\hline Body mass index $\left(\mathrm{kg} / \mathrm{m}^{2}\right)$ & $23.7 \pm 3.4$ & $22.6 \pm 3.9$ & 0.25 \\
\hline Diabetes mellitus & $16(59.3)$ & $14(53.8)$ & 0.69 \\
\hline Cerebrovascular disease & $10(37.0)$ & $4(15.4)$ & 0.07 \\
\hline Cardiovascular disease & $12(44.4)$ & $10(38.5)$ & 0.66 \\
\hline $\mathrm{CCl}$ & $2(2-3)$ & $3(2-3)$ & 0.14 \\
\hline \multicolumn{4}{|l|}{ Cause of KFRT } \\
\hline Diabetes mellitus & $16(59.3)$ & $12(46.2)$ & 0.46 \\
\hline Hypertension & 9 (33.3) & $10(38.5)$ & \\
\hline Glomerulonephritis & $1(3.7)$ & $3(11.5)$ & \\
\hline Others & $1(3.7)$ & $1(3.8)$ & \\
\hline BP-lowering drug & $23(85.2)$ & $21(80.8)$ & 0.67 \\
\hline ARB & $17(63.0)$ & $17(65.4)$ & 0.85 \\
\hline $\mathrm{CCB}$ & $11(40.7)$ & $14(53.8)$ & 0.34 \\
\hline$\beta$-Blocker & $12(44.4)$ & $15(57.7)$ & 0.34 \\
\hline a-Blocker & $3(11.1)$ & $6(23.1)$ & 0.25 \\
\hline $\mathrm{SBP}(\mathrm{mmHg})$ & $147.0 \pm 25.7$ & $148.1 \pm 22.6$ & 0.87 \\
\hline $\mathrm{DBP}(\mathrm{mmHg})$ & $73.5 \pm 17.4$ & $72.8 \pm 13.9$ & 0.86 \\
\hline Hemoglobin (g/dL) & $10.6 \pm 1.1$ & $10.2 \pm 1.3$ & 0.32 \\
\hline Albumin (g/dL) & $3.9 \pm 0.3$ & $3.9 \pm 0.3$ & 0.57 \\
\hline hs-CRP (mg/dL) & $0.7(0.11-1.4)$ & $0.8(0.23-1.5)$ & 0.87 \\
\hline Sodium (mmol/L) & $137.7 \pm 2.8$ & $134.0 \pm 17.7$ & 0.29 \\
\hline Potassium (mmol/L) & $5.1 \pm 0.7$ & $4.9 \pm 0.7$ & 0.35 \\
\hline Bicarbonate (mmol/L) & $23.6 \pm 4.6$ & $23.8 \pm 2.6$ & 0.89 \\
\hline PTH (pg/mL) & $255.5(188.7-395.6)$ & $265.8(169.6-451.9)$ & 0.75 \\
\hline Calcium (mg/dL) & $8.6 \pm 1.3$ & $8.9 \pm 0.6$ & 0.26 \\
\hline Phosphate (mg/dL) & $5.4 \pm 1.8$ & $5.2 \pm 1.3$ & 0.73 \\
\hline $\mathrm{BUN}(\mathrm{mg} / \mathrm{dL})$ & $62.1 \pm 15.8$ & $58.8 \pm 16.8$ & 0.47 \\
\hline Creatinine (mg/dL) & $10.1 \pm 2.4$ & $10.0 \pm 4.4$ & 0.88 \\
\hline $\mathrm{B} 2 \mathrm{MG}(\mathrm{mg} / \mathrm{L})$ & $21.7(19.7-23.4)$ & $25.6(21.4-28.0)$ & 0.006 \\
\hline Glucose (mg/dL) & $138.6 \pm 58.9$ & $132.2 \pm 73.2$ & 0.73 \\
\hline \multicolumn{4}{|l|}{ Dialysis parameter } \\
\hline Vintage (yr) & $1.5(1.0-4.25)$ & $3(1-4)$ & 0.89 \\
\hline Dialysis time (min) & $241.0 \pm 0.9$ & $241.6 \pm 2.5$ & 0.52 \\
\hline TMP (mmHg) & $150.2 \pm 47.6$ & $162.5 \pm 51.2$ & 0.37 \\
\hline Blood flow (mL/min) & $272.8 \pm 39.7$ & $256.2 \pm 31.8$ & 0.005 \\
\hline Heparin dose (IU/session) & $2,205.6(1,201.7-2,213.9)$ & 1,899.4 (1,603.3-2,209.5) & 0.48 \\
\hline Dialysate flow (mL/min) & $607.0 \pm 90.5$ & $589.5 \pm 113.3$ & 0.54 \\
\hline Net ultrafilation (L/sesseion) & $2.3 \pm 1.0$ & $2.3 \pm 1.1$ & 0.91 \\
\hline
\end{tabular}

Data are expressed as mean \pm standard deviation, number (\%), or median (interquartile range).

ARB, angiotensin receptor blocker; B2MG, $\beta 2$-microglobulin; BP, blood pressure; BUN, blood urea nitrogen; CCB, calcium channel blocker; CCI, Charlson comorbidity index; DBP, diastolic blood pressure; HDF, hemodiafiltration; hs-CRP, high-sensitivity C-reactive protein; KFRT, kidney failure with replacement therapy; PTH, parathyroid hormone; SBP, systolic blood pressure; TMP, transmembrane pressure.

$(25.4 \pm 5.6 \mathrm{mg} / \mathrm{L}$ to $22.2 \pm 4.4 \mathrm{mg} / \mathrm{L})$, while they remained relatively constant in the predilution HDF group at the same time period ( $\mathrm{p}$ for intergroup difference $=0.02)(\mathrm{Ta}-$ ble 3, Supplementary Fig. 2A). The $\beta 2$-microglobulin RRs 
Table 2. Primary outcome analysis: the differences in convection volume between predilution HDF and mixed-HDF

\begin{tabular}{|c|c|c|c|c|c|c|c|}
\hline \multirow[b]{2}{*}{ Variable } & \multirow[b]{2}{*}{ Treatment } & \multicolumn{4}{|c|}{ Observed data } & \multicolumn{2}{|c|}{ p-value } \\
\hline & & $\begin{array}{l}\text { Mean value } \\
\text { during } 6 \text { mo }\end{array}$ & Baseline & Month 3 & Month 6 & $\begin{array}{l}\text { Between- } \\
\text { group }\end{array}$ & $\begin{array}{l}\text { Within- } \\
\text { group }\end{array}$ \\
\hline \multicolumn{8}{|l|}{ Convection volume (L/session) } \\
\hline \multirow[t]{2}{*}{ Absolute } & Predilution & $41.0 \pm 10.3$ & $40.4 \pm 8.0$ & $39.1 \pm 8.7$ & $44.0 \pm 13.1$ & & 0.17 \\
\hline & Mixed & $35.0 \pm 5.7$ & $32.6 \pm 7.8$ & $36.3 \pm 4.2$ & $36.1 \pm 3.7$ & $<0.001$ & 0.01 \\
\hline \multirow[t]{2}{*}{ Effective } & Predilution & $41.0 \pm 10.3$ & $40.4 \pm 8.0$ & $39.1 \pm 8.7$ & $44.0 \pm 13.1$ & & 0.17 \\
\hline & Mixed & $51.5 \pm 9.0$ & $47.9 \pm 10.4$ & $53.3 \pm 7.6$ & $53.2 \pm 7.4$ & $<0.001$ & 0.003 \\
\hline \multicolumn{8}{|l|}{ Substitution volume (L/session) } \\
\hline \multirow[t]{2}{*}{ Via predilution mode } & Predilution & $38.8 \pm 10.5$ & $37.7 \pm 8.0$ & $36.8 \pm 8.9$ & $41.8 \pm 13.4$ & & 0.16 \\
\hline & Mixed & $16.6 \pm 4.2$ & $15.6 \pm 6.8$ & $17.1 \pm 2.3$ & $17.1 \pm 2.1$ & & 0.44 \\
\hline \multirow[t]{2}{*}{ Via postdilution mode } & Predilution & NA & NA & NA & NA & & \\
\hline & Mixed & $16.3 \pm 2.5$ & $14.8 \pm 2.6$ & $16.9 \pm 2.2$ & $17.1 \pm 2.1$ & & $<0.001$ \\
\hline \multirow[t]{2}{*}{ Net ultrafiltration (L/session) } & Predilution & $2.3 \pm 1.1$ & $2.3 \pm 1.0$ & $2.3 \pm 1.1$ & $2.2 \pm 1.1$ & & 0.72 \\
\hline & Mixed & $2.2 \pm 1.1$ & $2.3 \pm 1.1$ & $2.3 \pm 1.0$ & $1.9 \pm 1.1$ & 0.73 & 0.06 \\
\hline \multicolumn{8}{|c|}{$\begin{array}{l}\text { Convection volumes adjusted for } \\
\text { body surface area }\left(\mathrm{L} / \mathrm{m}^{2} \text { per session) }\right.\end{array}$} \\
\hline \multirow[t]{2}{*}{ Absolute } & Predilution & $24.5 \pm 3.8$ & $24.4 \pm 5.1$ & $23.1 \pm 5.5$ & $25.8 \pm 7.8$ & & 0.27 \\
\hline & Mixed & $20.6 \pm 5.5$ & $18.0 \pm 5.1$ & $20.0 \pm 4.1$ & $23.8 \pm 10.5$ & 0.004 & 0.004 \\
\hline \multirow[t]{2}{*}{ Effective } & Predilution & $24.5 \pm 3.8$ & $24.4 \pm 5.1$ & $23.1 \pm 5.5$ & $25.8 \pm 7.8$ & & 0.60 \\
\hline & Mixed & $31.5 \pm 5.3$ & $27.5 \pm 5.4$ & $32.6 \pm 4.6$ & $34.4 \pm 11.2$ & $<0.001$ & 0.003 \\
\hline
\end{tabular}

Data are presented as mean \pm standard deviation.

All analyses were conducted using repeated measures analysis of variance. HDF, hemodiafiltration; NA, not available.

at baseline did not differ between the two groups $(75.2 \% \pm$ $4.9 \%$ in the predilution HDF group vs. $76.0 \% \pm 4.8 \%$ in the mixed-HDF group). The RR significantly decreased in the predilution HDF group at 6 months, whereas it increased in the mixed-HDF group ( $p$ for intergroup difference $=0.01$ ) (Table 3, Fig. 3). Kt/V urea values, used as a traditional index for small-molecule clearance, were similar between the two groups throughout the study period (Table 3, Supplementary Fig. 2B). We also monitored TMP level during dialysis sessions because the mixed-HDF technique involves switching of HDF mode based on TMP level. The mixed-HDF group had consistently higher TMP level compared to the predilution HDF group during the 6-month study period ( $\mathrm{p}$ for intergroup difference $=0.001)($ Table 3, Supplementary Fig. 2C).

During the study period, there were no significant between-group differences in terms of serum hs-CRP, albumin, phosphate, sodium, potassium, and bicarbonate concentrations (Table 3). Out of 53 patients, 47 had available bioimpedance analysis data. As shown in Table 3, the proportions of overhydration status did not differ between the groups.

At baseline, SBP for the predilution HDF and mixed-HDF groups were $147 \pm 25.7$ and $148.1 \pm 22.6 \mathrm{mmHg}$, respectively. SBP increased at 3 months relative to baseline but then decreased at 6 months relative to the increase observed at 3 months in both groups. The difference observed between the two groups did not reach statistical significance. DBP of both groups remained similar throughout the 6-month study period (Table 3). Most patients tolerated HDF therapy well, and the symptomatic adverse event rate was remarkably low. The most common adverse effect observed was an asymptomatic decrease in SBP by $>10 \mathrm{mmHg}$ during a dialysis session, which occurred in $44 \%$ of all patients. However, symptomatic intradialytic hypotension was rarely observed in either group (3.7\% versus $1.3 \%$ in predilution and mixedHDF, respectively). There were no differences in any other event rates observed between the two groups (Table 4).

\section{Discussion}

In this randomized controlled study, we demonstrated that mixed-HDF therapy produces outcomes similar to predilution HDF. Convective volume was delivered well in the mixed-HDF group, and the difference in effective convec- 


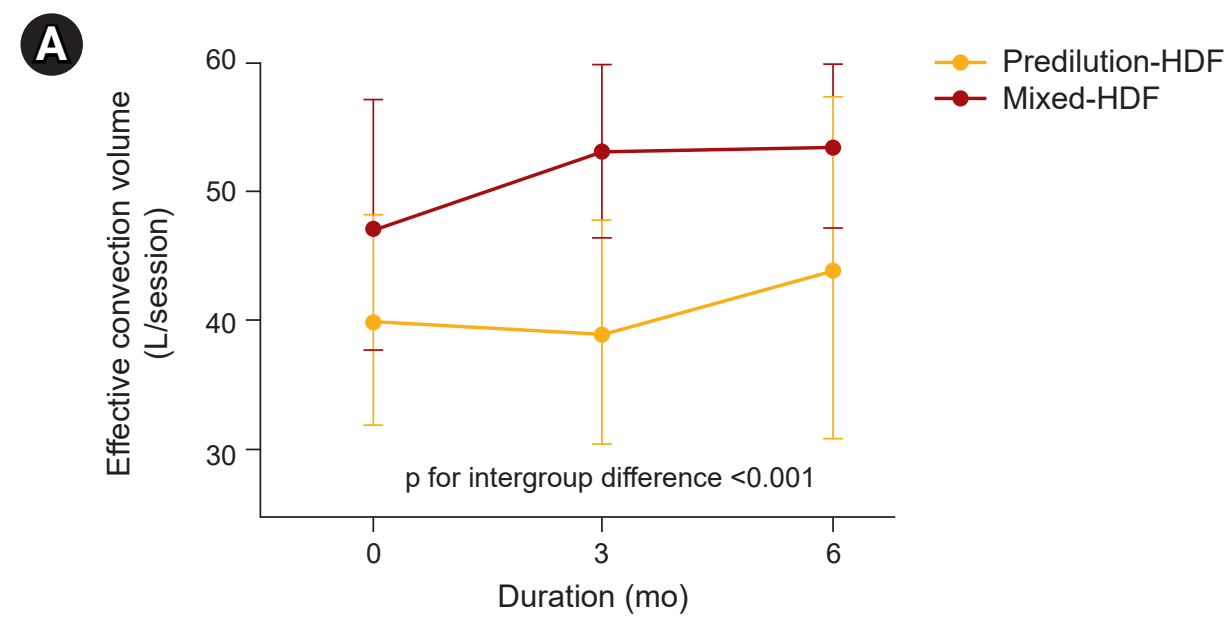

B

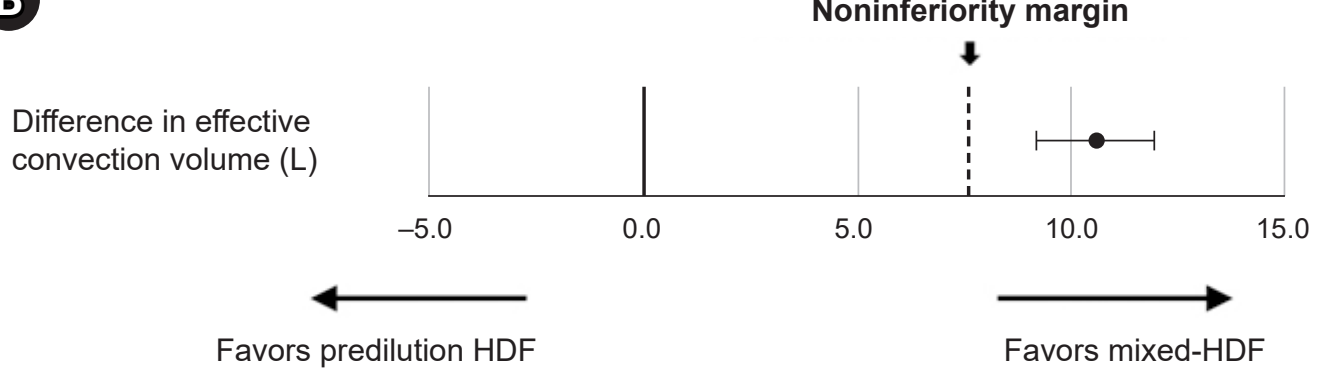

Figure 2. Changes and difference in effective convection volume. (A) Effective convection volume over 6 months ( $p$ for intergroup difference < 0.001). (B) Noninferiority of mixed-HDF compared with predilution HDF. The gray line indicates predilution HDF, and the black line indicates mixed-HDF.

HDF, hemodiafiltration.

tion volume met noninferiority criteria. We also showed that mixed-HDF more efficiently decreased $\beta 2$-microglobulin circulating level compared with predilution HDF. There were no differences in removal rates of small molecules, nor were other clinical or biochemical parameter differences observed during the study period. All adverse events were minor and occurred similarly in the two groups. These findings suggest that mixed-HDF is comparable to predilution HDF with regard to convection volume delivery, and that it removes middle molecules more efficiently.

HDF has been established as effective dialysis therapy, and its use has many advantages over conventional HD. These include higher clearance of middle molecular weight uremic toxins, better maintenance of hemodynamic stability, greater removal of inflammatory cytokines, and better responsiveness to erythropoietin $[19,20]$. Several randomized controlled trials have demonstrated that HDF reduces all-cause mortality, and even cardiovascular mortality, com-

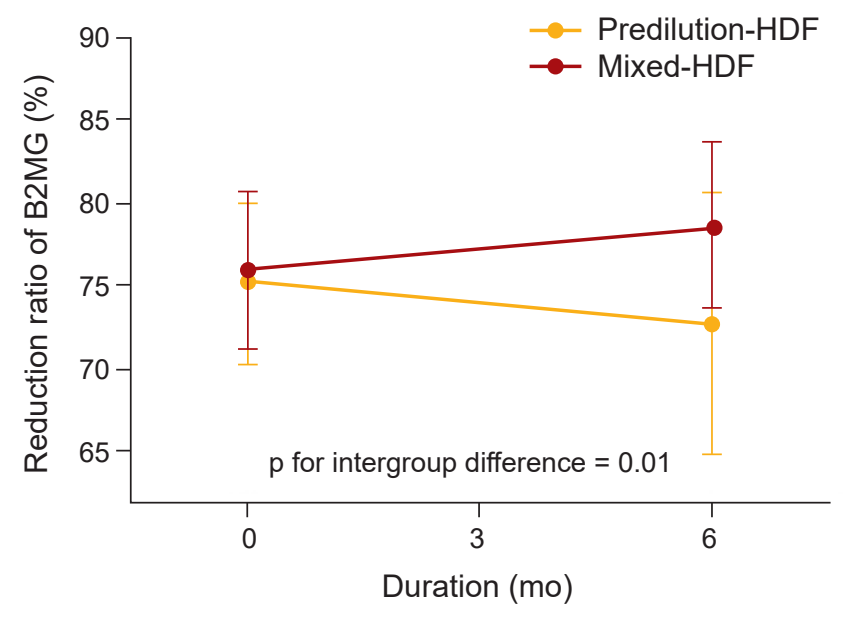

Figure 3. Changes in reduction ratios of B2MG during the study period ( $p$ for intergroup difference $=\mathbf{0 . 0 1}$ ). The yellow line indicates predilution HDF, and the red line indicates mixed-HDF. HDF, hemodiafiltration; B2MG, $\beta 2$-microglobulin. 
Table 3. Secondary outcome analysis

\begin{tabular}{|c|c|c|c|c|c|c|c|}
\hline \multirow[b]{2}{*}{ Variable } & \multirow[b]{2}{*}{ Treatment } & \multicolumn{4}{|c|}{ Observed data } & \multicolumn{2}{|c|}{ p-value } \\
\hline & & $\begin{array}{l}\text { Mean value of } \\
\text { three time points }\end{array}$ & Baseline & Month 3 & Month 6 & $\begin{array}{l}\text { Between- } \\
\text { group }\end{array}$ & $\begin{array}{l}\text { Within- } \\
\text { group }\end{array}$ \\
\hline \multirow[t]{2}{*}{ Predialysis B2MG (mg/L) } & Predilution & $21.2 \pm 4.8$ & $21.5 \pm 3.5$ & $21.2 \pm 6.3$ & $20.9 \pm 4.5$ & & 0.88 \\
\hline & Mixed & $23.6 \pm 4.7$ & $25.4 \pm 5.6$ & $23.3 \pm 3.6$ & $22.2 \pm 4.4$ & 0.02 & 0.004 \\
\hline \multirow[t]{2}{*}{ Reduction ratio of B2MG (\%) } & Predilution & $73.9 \pm 6.6$ & $75.2 \pm 4.9$ & NA & $72.8 \pm 7.9$ & & 0.36 \\
\hline & Mixed & $76.5 \pm 5.9$ & $76.0 \pm 4.8$ & NA & $78.7 \pm 5.1$ & 0.01 & 0.02 \\
\hline \multirow[t]{2}{*}{$\mathrm{Kt} / \mathrm{V}$ urea } & Predilution & $1.53 \pm 0.2$ & $1.53 \pm 0.3$ & $1.49 \pm 0.2$ & $1.57 \pm 0.2$ & & 0.37 \\
\hline & Mixed & $1.56 \pm 0.3$ & $1.51 \pm 0.3$ & $1.59 \pm 0.25$ & $1.57 \pm 0.3$ & 0.33 & 0.19 \\
\hline \multirow[t]{2}{*}{ TMP (mmHg) } & Predilution & $139.6 \pm 42.0$ & $150.2 \pm 47.6$ & $135.7 \pm 36.9$ & $132.9 \pm 40.3$ & & 0.09 \\
\hline & Mixed & $176.2 \pm 51.9$ & $162.5 \pm 51.2$ & $192.7 \pm 42.4$ & $173.1 \pm 59.1$ & 0.001 & 0.06 \\
\hline \multirow[t]{2}{*}{ hs-CRP (mg/dL) } & Predilution & $0.6(0.1-1.7)$ & $0.7(0.1-1.4)$ & $0.6(0.2-2.2)$ & $0.6(0.1-3.3)$ & & 0.14 \\
\hline & Mixed & $0.9(0.3-2.0)$ & $0.8(0.2-1.5)$ & $1.1(0.3-2.0)$ & $0.9(0.3-3.5)$ & 0.82 & 0.82 \\
\hline \multirow[t]{2}{*}{ Albumin (g/dL) } & Predilution & $3.9 \pm 0.3$ & $3.9 \pm 0.3$ & $4.0 \pm 0.4$ & $3.9 \pm 0.3$ & & 0.14 \\
\hline & Mixed & $3.8 \pm 0.4$ & $3.9 \pm 0.3$ & $3.8 \pm 0.4$ & $3.8 \pm 0.4$ & 0.16 & 0.14 \\
\hline \multirow[t]{2}{*}{ Phosphate (mg/dL) } & Predilution & $5.1 \pm 1.7$ & $5.4 \pm 1.8$ & $5.0 \pm 1.4$ & $4.8 \pm 1.7$ & & 0.30 \\
\hline & Mixed & $5.0 \pm 1.4$ & $5.2 \pm 1.3$ & $4.9 \pm 1.5$ & $4.8 \pm 1.3$ & 0.79 & 0.29 \\
\hline \multirow[t]{2}{*}{ Sodium (mmol/L) } & Predilution & $137.7 \pm 4.2$ & $137.7 \pm 2.9$ & $138.3 \pm 3.1$ & $137.2 \pm 6.0$ & & 0.51 \\
\hline & Mixed & $137.3 \pm 3.5$ & $137.9 \pm 4.3$ & $136.7 \pm 3.3$ & $137.3 \pm 2.7$ & 0.59 & 0.19 \\
\hline \multirow[t]{2}{*}{ Potassium (mmol/L) } & Predilution & $5.0 \pm 0.8$ & $5.1 \pm 0.7$ & $5.0 \pm 0.8$ & $4.9 \pm 0.8$ & & 0.49 \\
\hline & Mixed & $5.0 \pm 0.7$ & $4.9 \pm 0.7$ & $4.9 \pm 0.8$ & $5.2 \pm 0.6$ & 0.91 & 0.13 \\
\hline \multirow[t]{2}{*}{ Bicarbonate (mmol/L) } & Predilution & $23.4 \pm 4.0$ & $23.6 \pm 4.6$ & $23.4 \pm 3.5$ & $23.3 \pm 3.9$ & & 0.81 \\
\hline & Mixed & $23.4 \pm 3.0$ & $23.8 \pm 2.6$ & $23.4 \pm 3.7$ & $22.9 \pm 2.8$ & 0.89 & 0.13 \\
\hline \multicolumn{8}{|l|}{ Bioimpedance parameter } \\
\hline \multirow[t]{2}{*}{$\mathrm{OH}(\mathrm{L})$} & Predilution & & $0.8(-0.9$ to 1.2$)$ & NA & $1.0(-0.3$ to 2.7$)$ & & $>0.99$ \\
\hline & Mixed & & $0.3(-0.2$ to 1.23$)$ & NA & $0.7(-0.6$ to 2.9$)$ & 0.32 & 0.16 \\
\hline \multirow[t]{2}{*}{$\mathrm{OH}-\mathrm{ECW}(\%)$} & Predilution & & $4.5(-5.6$ to 9.1$)$ & NA & $7.1(-1.9$ to 14.6$)$ & & 0.98 \\
\hline & Mixed & & $2.7(-1.2$ to 8.0$)$ & NA & $4.1(-4.5$ to 18.2$)$ & 0.33 & 0.16 \\
\hline \multicolumn{8}{|c|}{ Predialysis blood pressure (mmHg) } \\
\hline \multirow[t]{2}{*}{ SBP } & Predilution & & $147.0 \pm 25.7$ & $155.8 \pm 25.5$ & $149.2 \pm 26.0$ & & 0.14 \\
\hline & Mixed & & $148.1 \pm 22.6$ & $156.1 \pm 24.7$ & $153.9 \pm 28.8$ & 0.74 & 0.22 \\
\hline \multirow[t]{2}{*}{ DBP } & Predilution & & $73.5 \pm 17.2$ & $74.0 \pm 14.6$ & $73.9 \pm 18.6$ & & $>0.99$ \\
\hline & Mixed & & $72.8 \pm 13.9$ & $74.9 \pm 15.9$ & $74.1 \pm 13.4$ & 0.97 & 0.68 \\
\hline
\end{tabular}

Data are presented as mean \pm standard deviation or median (interquartile range).

B2MG, $\beta 2$-microglobulin; DBP, diastolic blood pressure; hs-CRP, high-sensitive C-reactive protein; NA, not available; OH-ECW, overhydration/extracellular water; $\mathrm{OH}$, overhydration; SBP, systolic blood pressure; TMP, transmembrane pressure.

All analyses were conducted using repeated measures analysis of variance.

pared with conventional HD when an optimal convective substitution fluid was used [21-25]. Such proven benefits have resulted in the increased utilization of HDF in European countries [26]. There have been remarkable advances in HDF techniques, and several HDF methods have been implemented in clinical practice, including predilution, postdilution, and mixed modes. Most trials to date have tested the effects of postdilution HDF, and studies on other HDF modes are lacking. Interestingly, in East Asian countries, predilution HDF with a low access blood flow rate has been widely used [8]. A recently published observational study in Japan, where predilution HDF is preferred in most centers, suggested that this HDF mode was significantly associated with improved overall and cardiovascular survival compared with conventional HD [27].

Both pre- and postdilution HDF modes have pros and cons. Although the postdilution HDF mode, which is being recommended to most patients in Western countries, is associated with a highly efficient clearance of uremic toxins with a relatively small substitution volume, the limitation of 
Table 4. Adverse event rates

\begin{tabular}{|c|c|c|c|}
\hline Variable & Predilution HDF $(n=81)$ & Mixed-HDF $(n=78)$ & p-value \\
\hline No. of adverse events & $42(51.9)$ & $40(51.3)$ & 0.94 \\
\hline \multicolumn{4}{|l|}{ At least one event } \\
\hline A decrease in SBP $>10 \mathrm{mmHg}$ without symptoms & $36(44.4)$ & $34(43.6)$ & 0.73 \\
\hline Symptomatic intradialytic hypotension ${ }^{a}$ & $3(3.7)$ & $1(1.3)$ & \\
\hline Headache & $1(1.2)$ & $2(2.6)$ & \\
\hline Muscle cramps & $2(2.5)$ & $1(1.3)$ & \\
\hline Nausea, vomiting & 0 & $1(1.3)$ & \\
\hline Fever & 0 & $1(1.3)$ & \\
\hline Chest pain & 0 & 0 & \\
\hline Arrhythmia & 0 & 0 & \\
\hline
\end{tabular}

Data are presented as number (\%). The percentages were calculated with number of adverse events per dialysis sessions during study period. HDF, hemodiafiltration; SBP, systolic blood pressure.

aSymptomatic intradialytic hypotension is defined as a decrease in SBP by $\geq 20 \mathrm{mmHg}$ associated with symptoms that include abdominal discomfort, yawning, sighing, nausea, vomiting, muscle cramps, restlessness, dizziness or fainting, and anxiety.

the method is that blood flow should be maintained at a certain speed to reduce risk of blood clot formation. Patients in East Asian countries, who have relatively low blood flow of arteriovenous access, prefer predilution HDF mode to postdilution HDF, due to lower risk of clotting events in the former mode [6]. Recently, a new mode called mixed-HDF was developed to address the drawbacks of pre- and postdilution HDF. Mixed-HDF monitors optimal TMP and automatically switches to pre- or postdilution mode when the TMP level reaches a particular threshold, before a clotting issue occurs $[15,28]$. This process can compensate for the weaknesses of each HDF mode. Although few studies have compared the efficacy of mixed-HDF versus other HDF modes, Pedrini and De Cristofaro [2] reported that mixed-HDF had a better $\beta 2$-microglobulin removal rate than postdilution HDF. In addition, de Sequera et al. [29] showed that mixed-HDF was comparable to postdilution HDF with regard to small and medium-sized and protein-bound molecule clearance.

Delivered convective volume is considered a crucial factor that influences HDF therapy. Mixed-HDF is a new concept of high-efficiency HDF in which predilution and postdilution modes are mixed, and it is difficult to compare quantitatively absolute substitution fluid with those of the predilution mode. Given that a greater volume of substitution fluid is required in predilution mode versus postdilution HDF mode, we developed the concept of using effective convection volume for quantitative comparison between the two groups. Since the predilution mode of HDF uses twice as much replacement fluid as does postdilution mode, we hypothesized that the effective convection volume via mixed-HDF mode consisted of a sum of the substitution volume in postdilution mode times two, substitution volume in predilution mode, and ultrafiltration volume. Although the absolute convection volume was higher in predilution HDF, we showed that the effective convection volume delivered by mixed-HDF was greater than that of predilution HDF. The effective convection volume from mixed-HDF was approximately $20 \%$ higher than that of predilution HDF. The optimal convection volume delivered by mixed-HDF remains unknown because no studies have yet examined mixedHDF outcomes such as mortality or cardiovascular events based on convection volume. In this regard, the concept of effective convection volume might be an alternative tool for the comparison of convection volumes for predilution and mixed-HDF. Notably, differences in effective convection volume might result in improved $\beta 2$-microglobulin clearance by mixed-HDF. This is important because the accumulation of middle molecules, such as $\beta 2$-microglobulin, is an independent predictor of mortality [30-32], and $\beta 2$-microglobulin clearance correlates well with convection volume [33]. In addition, dialysis tolerance of patients was similar for the two HDF modes. All adverse events were minor, and the event rates of both groups were similar. Taken together, these findings suggest that mixed-HDF can be an alternative dialysis tool for patients who frequently experience dialysis-related symptoms such as intradialysis hypotension, muscle cramping, and arrhythmia [25].

This study has limitations. First, the sample size was small, 
and only two centers participated. Therefore, selection bias could not be excluded. Second, we could not determine optimal convection volume. It should be noted that many previous trials with postdilution HDF consistently showed improved patient survival rate compared with conventional HD in patients with adequately delivered convection volume. To date, there has been no randomized controlled trial that has assessed the delivered convection volume in patients given predilution HDF or mixed-HDF therapy. Future studies with larger sample sizes will be needed to address this. Third, we measured $\beta 2$-microglobulin and hs-CRP levels as representative middle molecule and inflammatory markers, although other uremic toxins with deleterious effects exist. Because the predilution mode has an intrinsic limitation in the degree of clearance compared with postdilution HDF, the performance of mixed-HDF should be further tested using other molecules. Fourth, we used a simple equation of RR of $\beta 2$-microglobulin, which was not calibrated for body fluid reduction during dialysis. However, as there was no significant difference in ultrafiltration volume between the predilution and mixed-HDF groups, ultrafiltration volume is unlikely to alter the outcomes. Finally, we did not evaluate albumin loss via dialyzer during the study. Albumin loss via HDF can differ depending on the dialyzer, dialysis modalities, and convection volumes. Previous studies have reported a wide range of albumin loss between 0.5 and $4.5 \mathrm{~g}$ per session in postdilution HDF [34-36]. The amount of albumin loss in mixed-HDF is not well known. Although albumin loss is less severe in predilution HDF than in postdilution HDF, albumin loss via HDF can be especially important for patients with marked hypoalbuminemia. However, in this study, the two groups used the same dialyzer that has a very low albumin-sieving coefficient according to the manufacturer. Moreover, serum albumin levels were comparable between the two groups. Thus, albumin loss was unlikely to affect the study results.

In conclusion, we demonstrated that mixed-HDF performed well with regard to convective volume delivery and provided better middle molecule clearance than predilution HDF. For the implementation of mixed-HDF in clinical practice, further studies should explore whether the use of mixed-HDF is advantageous over other dialysis modes with regard to its cost-effectiveness and long-term outcomes.

\section{Conflicts of interest}

Tae-Hyun Yoo is the Editor-in-Chief of Kidney Research and Clinical Practice and was not involved in the review process of this article. All authors have no other conflicts of interest to declare.

\section{Funding}

This study was supported by Fresenius Medical Care Korea.

\section{Authors' contributions}

Conceptualization: SHH

Data collection and curation: All authors

Formal analysis: KSP

Funding acquisition: $\mathrm{SHH}$

Investigation: KSP, WJ

Writing - original draft: KSP

Writing - review \& editing: SHH

All authors read and approved the final manuscript.

\section{ORCID}

Kyoung Sook Park, https://orcid.org/0000-0002-6365-0428 Ea Wha Kang, https://orcid.org/0000-0002-2153-068X Tae Ik Chang, https://orcid.org/0000-0003-3311-6379 Wonji Jo, https://orcid.org/0000-0002-8685-2516 Jung Tak Park, https://orcid.org/0000-0002-2325-8982 Tae-Hyun Yoo, https://orcid.org/0000-0002-9183-4507 Shin-Wook Kang, https://orcid.org/0000-0002-5677-4756 Seung Hyeok Han, https://orcid.org/0000-0001-7923-5635

\section{References}

1. Saran R, Robinson B, Abbott KC, et al. US Renal Data System 2018 Annual Data Report: Epidemiology of Kidney Disease in the United States. Am J Kidney Dis 2019;73(3 Suppl 1):A7-A8.

2. Pedrini LA, De Cristofaro V. On-line mixed hemodiafiltration with a feedback for ultrafiltration control: effect on middle-molecule removal. Kidney Int 2003;64:1505-1513.

3. Jirka T, Cesare S, Di Benedetto A, et al. Mortality risk for patients receiving hemodiafiltration versus hemodialysis. Kidney Int 2006;70:1524.

4. Pedrini LA, Cozzi G, Faranna P, et al. Transmembrane pres- 
sure modulation in high-volume mixed hemodiafiltration to optimize efficiency and minimize protein loss. Kidney Int 2006;69:573-579.

5. Park KW, Kyun Bae S, Lee B, et al. The effect of on-line hemodiafiltration on heart rate variability in end-stage renal disease. Kidney Res Clin Pract 2013;32:127-133.

6. Ahrenholz P, Winkler RE, Ramlow W, Tiess M, Müller W. On-line hemodiafiltration with pre- and postdilution: a comparison of efficacy. Int J Artif Organs 1997;20:81-90.

7. Wizemann V, Külz M, Techert F, Nederlof B. Efficacy of haemodiafiltration. Nephrol Dial Transplant 2001;16 Suppl 4:27-30.

8. Schiffl H. Online hemodiafiltration and mortality risk in endstage renal disease patients: a critical appraisal of current evidence. Kidney Res Clin Pract 2019;38:159-168.

9. Tsuchida K, Minakuchi J. Clinical benefits of predilution on-line hemodiafiltration. Blood Purif 2013;35 Suppl 1:18-22.

10. Canaud B, Lévesque R, Krieter D, et al. On-line hemodiafiltration as routine treatment of end-stage renal failure: why pre- or mixed dilution mode is necessary in on-line hemodiafiltration today? Blood Purif2004;22 Suppl 2:40-48.

11. Masakane I, Kikuchi K, Kawanishi H. Evidence for the clinical advantages of predilution on-line hemodiafiltration. Contrib Nephrol 2017;189:17-23.

12. Kim ST, Yamamoto C, Taoka M, Takasugi M. Programmed filtration, a new method for removing large molecules and regulating albumin leakage during hemodiafiltration treatment. Am J Kidney Dis 2001;38(4 Suppl 1):S220-S223.

13. Pedrini LA, De Cristofaro V, Pagliari B, Samà F. Mixed predilution and postdilution online hemodiafiltration compared with the traditional infusion modes. Kidney Int 2000;58:2155-2165.

14. Feliciani A, Riva MA, Zerbi S, et al. New strategies in haemodiafiltration (HDF): prospective comparative analysis between online mixed HDF and mid-dilution HDF. Nephrol Dial Transplant 2007;22:1672-1679.

15. Pedrini LA, Zerbi S. Mixed-dilution hemodiafiltration. Contrib Nephrol 2007;158:123-130.

16. Akizawa T, Koiwa F. Clinical expectation of online hemodiafiltration: a Japanese perspective. Blood Purif 2015;40 Suppl 1:12-16.

17. Tattersall JE, Ward RA; EUDIAL group. Online haemodiafiltration: definition, dose quantification and safety revisited. Nephrol Dial Transplant 2013;28:542-550.

18. Potier J, Bowry S, Canaud B. Clinical performance assessment of CorDiax filters in hemodialysis and hemodiafiltration. Contrib Nephrol 2017;189:237-245.

19. Vaslaki L, Major L, Berta K, et al. On-line haemodiafiltration ver- sus haemodialysis: stable haematocrit with less erythropoietin and improvement of other relevant blood parameters. Blood Purif 2006;24:163-173.

20. Pedrini LA, Zawada AM, Winter AC, et al. Effects of high-volume online mixed-hemodiafiltration on anemia management in dialysis patients. PLoS One 2019;14:e212795.

21. Canaud B, Vienken J, Ash S, Ward RA; Kidney Health Initiative HDF Workgroup. Hemodiafiltration to address unmet medical needs ESKD patients. Clin J Am Soc Nephrol 2018;13:1435-1443.

22. Blankestijn PJ, Grooteman MP, Nube MJ, Bots ML. Clinical evidence on haemodiafiltration. Nephrol Dial Transplant 2018; 33(Suppl 3):iii53-iii58.

23. Ok E, Asci G, Toz H, et al. Mortality and cardiovascular events in online haemodiafiltration (OL-HDF) compared with high-flux dialysis: results from the Turkish OL-HDF Study. Nephrol Dial Transplant 2013;28:192-202.

24. Maduell F, Moreso F, Pons M, et al. High-efficiency postdilution online hemodiafiltration reduces all-cause mortality in hemodialysis patients. J Am Soc Nephrol 2013;24:487-497.

25. Morena M, Jaussent A, Chalabi L, et al. Treatment tolerance and patient-reported outcomes favor online hemodiafiltration compared to high-flux hemodialysis in the elderly. Kidney Int 2017;91:1495-1509.

26. Sichart JM, Moeller S. Utilization of hemodiafiltration as treatment modality in renal replacement therapy for end-stage renal disease patients: a global perspective. Contrib Nephrol 2011;175:163-169.

27. Kikuchi K, Hamano T, Wada A, Nakai S, Masakane I. Predilution online hemodiafiltration is associated with improved survival compared with hemodialysis. Kidney Int 2019;95:929-938.

28. Pedrini LA. On-line hemodiafiltration: technique and efficiency. J Nephrol 2003;16 Suppl 7:S57-S63.

29. de Sequera P, Albalate M, Pérez-García R, et al. A comparison of the effectiveness of two online haemodiafiltration modalities: mixed versus post-dilution. Nefrologia 2013;33:779-787.

30. Wang AY, Ninomiya T, Al-Kahwa A, et al. Effect of hemodiafiltration or hemofiltration compared with hemodialysis on mortality and cardiovascular disease in chronic kidney failure: a systematic review and meta-analysis of randomized trials. Am J Kidney Dis 2014;63:968-978.

31. Foster MC, Coresh J, Hsu CY, et al. Serum $\beta$-trace protein and $\beta 2$-microglobulin as predictors of ESRD, mortality, and cardiovascular disease in adults with CKD in the Chronic Renal Insufficiency Cohort (CRIC) study. Am J Kidney Dis 2016;68:68-76.

32. Masakane I, Sakurai K. Current approaches to middle molecule removal: room for innovation. Nephrol Dial Transplant 
2018;33(Suppl 3):iii12-iii21.

33. Canaud B, Barbieri C, Marcelli D, et al. Optimal convection volume for improving patient outcomes in an international incident dialysis cohort treated with online hemodiafiltration. Kidney Int 2015;88:1108-1116.

34. Cuvelier C, Tintillier M, Migali G, Van Ende C, Pochet JM. Albumin losses during hemodiafiltration: all dialyzers are not created equal - a case report. BMC Nephrol 2019;20:392.

35. Lee YH, Shin YS, Lee SY, et al. Effects of online hemodiafiltration on anemia and nutritional status in chronic hemodialysis patients. Kidney Res Clin Pract 2020;39:103-111.

36. Fournier A, Birmelé B, François M, Prat L, Halimi JM. Factors associated with albumin loss in post-dilution hemodiafiltration and nutritional consequences. Int J ArtifOrgans 2015;38:76-82. 